\title{
Spin Hall effect originated from fractal surface
}

\author{
I. Hajzadeh ${ }^{1}$, S.M. Mohseni ${ }^{1, *}$, S.M.S. Movahed ${ }^{1}$, G.R. Jafari ${ }^{1}$ \\ ${ }^{1}$ Department of Physics, Shahid Beheshti University, Velenjak, Tehran 19839, Iran*
}

\begin{abstract}
Spin hall effect (SHE) in thin films is inherited by surface roughness. Although roughness effect on SHE has been studied in thin films, but roughness is not only parameter in rough surfaces. Our results show that how other statistical parameters of rough surface play important role in SHE. In this paper we investigate theoretically the effects of correlated surface roughness in the SHE with self affine fractal surface in non-heavy metallic thin films in the frame work of the Born approximation. The surface roughness is described by the k-correlation model and is characterized by the roughness exponent $H(0 \leq H \leq 1)$, the in plane correlation length $\xi$ and the rms roughness amplitude $\delta$. We show that the spin Hall angle can increase by one order of magnitude when $H$ decreasing from $H=1$ to $H=0$. We also demonstrate the SHE for surface roughness with distribution function of the Gaussian profile is mainly contributed by the side jump scattering while for that with a nonGaussian profile, both side jump and skew scattering are present. our achievements demonstrate the important role of roughness texture profile for SHE in non-heavy metals.
\end{abstract}




\section{INTRODUCTION}

Spin Hall effect (SHE) and its inverse effect (ISHE) are a group of phenomena emerge from spin-orbit coupling (SOC) in non-magnetic metals and semiconductors. SHE converts electrical conductivity to transverse spin Hall conductivity (SHC) in non-magnetic layer without using magnetic field (ISHE acts in opposite way exactly) ${ }^{1}$. vide a possibility of detection and generation of SHC in non-magnetic materials ${ }^{6 / 13}$ and have potential applications in spintronic devices, such as Spin-Hall oscillators, SHE transistors, spin photodetectors, spin thermoelectric converters, domain wall electronics and spin Hall magnetic memories, etc $\sqrt{3 \mid 12} \mid 22$.

A key challenge for the promotion of such spintronic devices is to attain efficient conversion between charge and spin currents. It has been believed that heavy metals with strong spin-orbit interaction are indispensable. This is largely restrictions for the selection of materials for the practical application of the spintronic devices. Light metals have been confirmed to exhibit negligible SHEs. Thus, whether the SHEs can be enhanced in light metals is an important fundamental and practical question to push forward the application of the spintronic devices with a large selection of materials. In this study, we demonstrate that light metals (Our analysis based on $\mathrm{Cu}$ thin film) becomes an efficient converter between spin and charge currents through correlated surface roughness and statistical parameters of rough surface. Recently, the problem of surface roughness with uncorrelated surface profile on the SHC of non-magnetic thin metallic films has been developed by Zhou et al ${ }^{23}$. But, an important question comes with "can uncorrelated roughness describe a real surface?". AFM images in thin film deposition show that thin film surfaces are correlated and self-affine ${ }^{24 \mid 25}$. Also, the height-height correlation function plays a significant role in the limit $k_{F} \xi \gg 1$, where $\xi$ is the in plane correlation length for the surface roughness and $k_{F}$ is the Fermi wave vector ${ }^{26}$. To address these facts, we focus on the correlated surface that have fractality effect.

In this paper, to accomplish the argument about effects of self-affinity in SHA in light metals, and effects of Gaussian and non-Gaussian of distribution functions of surface roughness in SHC, we explore the influence of the height-height correlation function on the SHA with self-affine surface roughness. The surface roughness will be regarded as effective impurities ${ }^{27 / 28}$ and the power spectrum of isotropic rough surface will be described by 
k-correlation model $29+32$. The self-affine fractal surface roughness is characterized in addition to height fluctuations $\delta$, from flatness and $\xi$ by a local fractal dimension $d_{f}=3-H$, where $H$ is the Hurts or roughness exponent and $0 \leq H \leq 1$. Smaller value of $H$ and $\xi$ correspond to the rougher surface. By introducing the spin-orbit interaction associated with fractal effective impurity, we find that the main interplay mechanism of the roughness effect on SHA occurs for $H$ and $\xi$, and SHA can increase by one order of magnitude, when the roughness exponent varies from $H=1$ to $H=0$. Moreover, we find that if the distribution function of surface roughness taken to be Gaussian, the SJ contributes to the SHE and the skew scattering (SS) does not have any contribution in SHC induced by surface roughness. Contrarily, for that to be non-Gaussian, the SS contributes in SHC.

The rest of this paper is organized as follows. In Sec.II, we present a theoretical description for a thin film with generalized correlated surface roughness and the uncorrelated bulk impurities. In Sec.III, using the transition probability and relaxation times, we obtain the longitudinal conductivity and the SHC. Furthermore, we describe the influence of Gaussian and non-Gaussian roughness distribution functions on SHE. In Sec.IV, a typical model for a self-affine rough surface characterized by power-law function for correlation are described. We also discuss the routes to enhance the surface roughness induced spin-orbit interaction due to the fractal surface scattering. Finally some general summary and conclusions are presented in Sec.V.

\section{MODEL}

We consider a metal thin film with correlated rough surface extended in a plane with $\rho=$ $(x, y)$ direction and confines in the $z$ direction with variable thickness $d(\rho)$. The confinement leads to discrete energy levels which depend on the film thickness. The total Hamiltonian is given by:

$$
\mathcal{H}=\mathcal{H}_{0}+\mathcal{H}_{1}
$$

The first term describes a film of constant thickness $d$, without roughness represented by:

$$
\mathcal{H}_{0}=\frac{|\vec{p}|^{2}}{2 m}+u_{d}(z)=\sum_{n q} \varepsilon_{n q} a_{n q \sigma}^{+} a_{n q \sigma}
$$


where $m$ is the mass of electron and $\vec{p}=i \hbar \vec{\nabla}$ is the momentum operator, $u_{d}(z)$ is the confined potential of a particle in a box. The right term in Eq. (2) is the kinetic energy of conduction electrons with energy $\varepsilon_{n q}=\hbar^{2} q^{2} / 2 m^{*}-\varepsilon_{F}$ measured from Fermi level $\varepsilon_{F}, q=\left(q_{x}, q_{y}\right)$ is in plane wave vector and $m^{*}$ is effective mass, where $\frac{1}{m^{*}}=\frac{1}{m}\left(\frac{k_{n}^{2}}{q^{2}}+1\right), k_{n}=n \pi / d$, and $n$ denotes transverse mode and the operator $a_{n q \sigma}^{+}\left(a_{n q \sigma}\right)$ creates (annihilates) an electron with spin $\sigma$ at $n$th level. The second term in Eq.(1) $\mathcal{H}_{1}$ becomes,

$$
\mathcal{H}_{1}=\sum_{n n^{\prime}} \sum_{q q^{\prime}} \sum_{\sigma \sigma^{\prime}}\left\langle n^{\prime} q^{\prime} \sigma^{\prime}|U| n q \sigma\right\rangle a_{n^{\prime} q^{\prime} \sigma^{\prime}}^{+} a_{n q \sigma}
$$

where $\langle\ldots\rangle$ denotes the expectation value over the state $|n q \sigma\rangle=\sqrt{2 / \mathcal{A d}} \sin \left(k_{n} z\right) \exp (i q . \rho)|\sigma\rangle$, $\mathcal{A}$ is the lateral area, $|\sigma\rangle$ is the eigenspin state with $\hat{\sigma}|\sigma\rangle=\sigma|\sigma\rangle$ and $\hat{\sigma}=\left(\sigma_{x}, \sigma_{y}, \sigma_{z}\right)$ is the Pauli spin operator. The total impurity potential $U$ is the sum of the bulk impurity $u_{I}$, surface roughness scattering $u_{R}$ and the spin-orbit interaction due to the surface scattering $u_{R}^{S O}, U=u_{I}+u_{R}+u_{R}^{S O}$. Summarizes, Eq.(3) is the scattering of conduction electrons between different transverse modes of momentum and spin states. Now we concentrate on scattering mechanism treated as perturbation and introduce three terms as original reasons for it. First of all, the bulk impurity scattering can be addressed with a short range impurity potential with concentration $n_{i m p}$,

$$
u_{I}(r)=\frac{u_{i m p}}{k_{F}^{3}} \sum_{i} \delta_{D}\left(r-r_{i}\right)
$$

where $\delta_{D}$ is Dirac delta function. The magnitude of the potential is given by $u_{i m p}, r_{i}=$ $\left(\rho_{i}, z_{i}\right)$ stands for the position of $i$ th impurity and $k_{F}$ is the Fermi wave vector. Next, we consider a rough surface, by a dilation operator with $\lambda_{\rho}=\ln \frac{d}{d(\rho)}$ the surface roughness is converted into effective scattering potential $27 / 28$.

$$
u_{R}=\lambda_{\rho}\left(2 u_{d}+z \partial z u_{d}\right)
$$

We assume that the volume of the original film with rough surface remains unchanged after dilation transformation so the ensemble average over roughness profile equal to $\langle d(\rho)\rangle=d$, and hence $\left\langle\lambda_{\rho}\right\rangle=0$. Furthermore, we consider the surface roughness is correlated and to be isotropic, $C\left(\rho=\left|\rho^{\prime}-\rho^{\prime \prime}\right|\right)=\left\langle\lambda_{\rho^{\prime}} \lambda_{\rho^{\prime \prime}}\right\rangle$. In addition, the gradient of the effective scattering 
potential leads to a $\mathrm{SOC}^{23}$, as

$$
u_{R}^{S O}=\frac{\eta}{\hbar} \sigma \cdot\left(\vec{p} \times \nabla u_{R}\right)
$$

where $\eta$ is the SOC parameter for the surface scattering and $\hbar$ is the reduced Planck's constant.

Considering Eqs. (4), (5) and (6), the second term in Eq. (1) becomes

$$
\begin{aligned}
\left\langle n^{\prime} q^{\prime} \sigma^{\prime}|U| n q \sigma\right\rangle & =u_{q^{\prime} q} \delta_{\sigma \sigma^{\prime}} \delta_{n n^{\prime}}+u_{R_{n^{\prime} q^{\prime} n q}} \delta_{\sigma \sigma^{\prime}} \delta_{n n^{\prime}} \\
& +i \eta u_{R_{n^{\prime} q^{\prime} n q}}\left[\sigma_{\sigma \sigma^{\prime}} \cdot\left(q^{\prime} \times q\right)\right] \delta_{n n^{\prime}}
\end{aligned}
$$

where $u_{q^{\prime} q}=\left\langle q^{\prime}\left|u_{I}\right| q\right\rangle=\left(u_{i m p} / V\right) \sum_{i} \exp \left[i\left(q-q^{\prime}\right) \cdot r_{i}\right], V$ is the volume of the film. $u_{R_{n^{\prime} q^{\prime} n q}}=\left\langle n^{\prime} q^{\prime}\left|u_{R}\right| n q\right\rangle=\lambda_{q-q^{\prime}} \varepsilon_{0} n n^{\prime}$ with $\varepsilon_{0}=\hbar^{2} \pi^{2} / 2 m d^{2}$ and $\lambda_{q-q^{\prime}}$ is the Fourier component of $\lambda_{\rho}$ of the wave vector $q-q^{\prime}$.

\section{CONDUCTIVITIES}

\section{A. Longitudinal conductivity}

Based on Lippman-Schwinger formalism we can calculate the transition probability, $P$ from state $|n q \sigma\rangle$ to $\left|n^{\prime} q^{\prime} \sigma^{\prime}\right\rangle$,

$$
P_{n q \sigma}^{n^{\prime} q^{\prime} \sigma^{\prime}}=\frac{2 \pi}{\hbar}\left|\left\langle n^{\prime} q^{\prime} \sigma^{\prime}|T| n q \sigma\right\rangle\right|^{2} \delta_{D}\left(\varepsilon_{n q \sigma}-\varepsilon_{n^{\prime} q^{\prime} \sigma^{\prime}}\right)
$$

where $T=U+U(E-\mathcal{H})^{-1} U$ is the scattering matrix, whose matrix elements are calculated up to the second-order Born approximation. The relaxation time allows us to compute the

conductivities. The relaxation rate for each channel at Fermi energy is obtained from the transition probability shown in Eq.(8), after ensemble averaging over surface profiles and employing Matthiessen's rule, we can find relaxation times. The total scattering rate is,

$$
\frac{1}{\tau_{n}}=\sum_{n^{\prime} q^{\prime} \sigma^{\prime}} P_{n q \sigma}^{n^{\prime} q^{\prime} \sigma^{\prime}}=\frac{1}{\tau_{0}}+\frac{1}{\tau_{n}^{\prime}}
$$




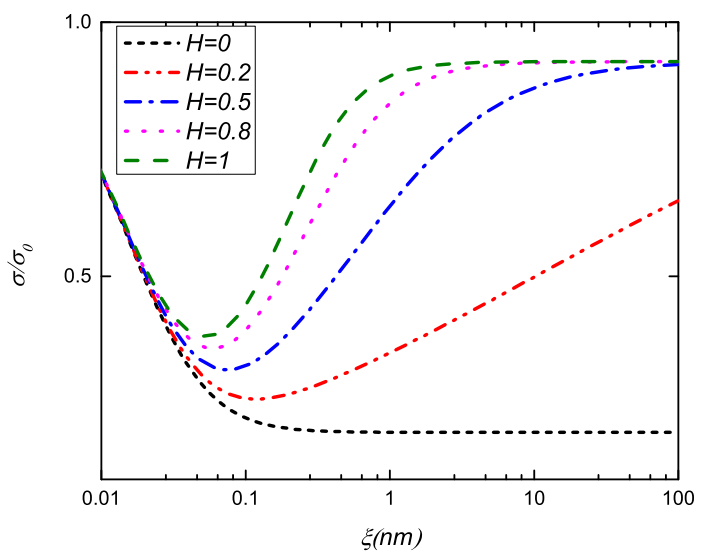

Figure 1. Longitudinal conductivity $\sigma$, for $C u$ film vs. correlation length $\xi$ for $\varepsilon_{F}=7 e V, \tau_{0}=24 f s$, $q=k_{F}=1.361 / \AA, n_{c}=10, \delta=5 a_{0}, a_{0}=3.61 \AA, \sigma_{0}=5.88 \times 10^{7} \mathrm{~s} / \mathrm{m}$, and $H$, as indicated.

where

$$
\frac{1}{\tau_{0}}=\frac{u_{i m p}^{2}}{\varepsilon_{F} \hbar} \frac{n_{i m p}}{2 \pi k_{F}^{3} n_{c}}\left(1+2 n_{c}\right)
$$

and

$$
\frac{1}{\tau_{n}^{\prime}}=\frac{2}{3} \frac{\varepsilon_{F}}{\hbar} \frac{\sum_{n=1}^{n_{c}} n^{2} / n_{c}^{3}}{n_{c}\left(\sum_{n=1}^{n_{c}} \frac{1}{n^{2}}-1\right)}\left\langle|S(q)|^{2}\right\rangle,
$$

where $\tau_{0}^{-1}$ is the bulk impurity scattering rate and $\tau_{n}^{\prime-1}$ is the channel (n) dependent surface scattering rate, $\left\langle|S(q)|^{2}\right\rangle$ is the Fourier transform $C(\rho)$ which is called power spectrum and the total number of transverse channels is $n_{c}=k_{F} d / \pi$ and $n \leq n_{c}$. In Eq.11 we show that $\tau_{n}^{\prime-1}$ is dependent to power spectrum or height-height correlation function therefore by changing of $C(\rho)$ we can make a difference in $\tau_{n}^{\prime-1}$ and optimize SHC and SHA.

Employing the aforementioned formalism results in longitudinal conductivity for any correlated surface roughness by 2728

$$
\sigma=\frac{3 \sigma_{0}}{2 n_{c}} \sum_{n=1}^{n c} \frac{\tau_{n}}{\tau_{0}}\left(1-\frac{n^{2}}{n_{c}^{2}}\right),
$$

where $\sigma_{0}=k_{F}^{3} e^{2} \tau_{0} / 3 \pi^{2} m^{*}$ is the bulk Drude conductivity. In Fig, 1 we present the room temperature longitudinal conductivity versus correlation length $\xi$ for $C u$ thin film, for several values of the roughness exponent $H$. There is a minimum in the film conductivity as a function of the correlation length, which occurs approximately at $\xi=0.1 \mathrm{~nm} \sim \mathcal{O}\left(a_{o}\right)$, 


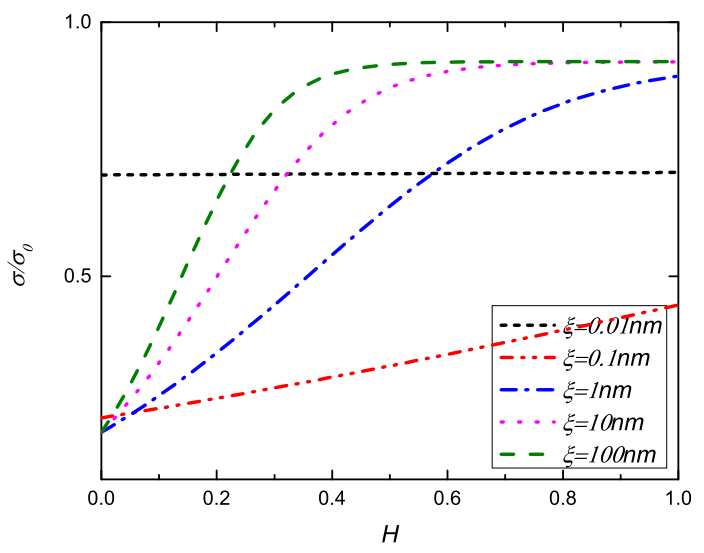

Figure 2. $\sigma$ as a function of the Hurst exponent $H$ with same parameters used in Fig 1 and indicated values of correlation length $\xi$.

where $a_{0}$ is the lattice constant. For $\xi>>0.1 \mathrm{~nm}$ the conductivity increases with increasing $H$ or increasing $\xi$ (smoother surface). For small values of $\xi<<0.1 \mathrm{~nm}$ the situation is reversed (in this case the correlation length is so smaller than the lattice constant, therefore we can suppose that the surface is uncorrelated $\xi \sim 0)$. This is due to the fact that this kind of roughness does not scatter electrons when their wavelength is much longer than the correlation length $\xi$. For large values of $\xi$ the behavior is more complex, i.e., the conductivity reaches to a maximum with strong dependency on $H$. Fig, 2 shows the dependence of the film conductivity on the roughness exponent $H$ shown for several values of the correlation length $\xi$. For $\xi=10,100 \mathrm{~nm}$, the conductivity first increases with increasing $H$, with a further increase of the roughness exponent $H$. For the correlation lengths longer than $a_{0}$ the conductivity increases with increasing $\xi$ at a much faster rate for large $H(H \sim$ 1). Additionally, with larger correlation length this maximum point shifts to the smaller roughness exponent. For the extreme limit, as can be seen from the curve that correspond to the logarithmic roughness $(H=0)$ in Fig.2, the conductivity increases extremely slowly with increasing correlation length. Thus, the smoothing effect at large length scale is strongly influenced by the roughness exponent. The result agrees with those obtained in Ref 29 . 


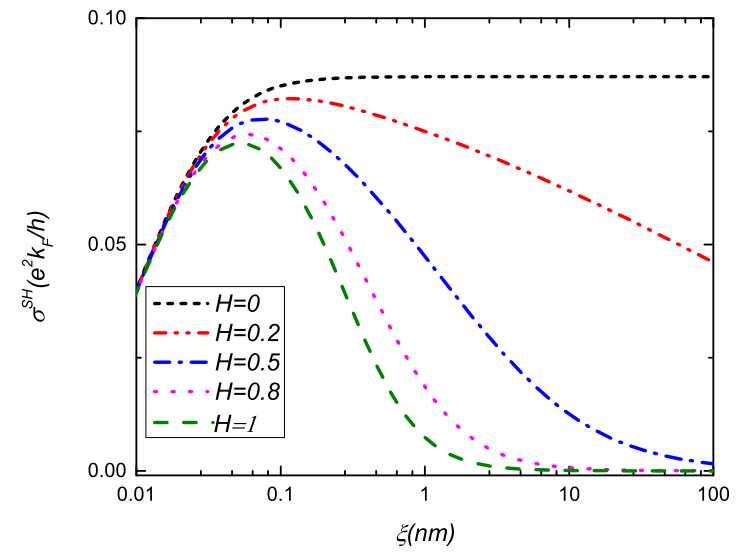

Figure 3. SHC $\sigma^{S H}$ vs. correlation length $\xi$ for $C u$ film with same parameters used in Fig 1 , and $\bar{\eta}=0.5$ and indicated values of $H$.

\section{B. Spin Hall conductivity}

SHE originates from three mechanisms, the SS, SJ and intrinsic ${ }^{6}$,

$$
\sigma^{S H}=\alpha_{s s}^{S H} \sigma+\alpha_{s j}^{S H} \sigma+\sigma_{i n t}^{S H},
$$

here $\alpha_{s s}^{S H}$, is the SHA from skew mechanism. If roughness scattering potential distribution function is symmetrical, it means that the third moment or skewness of scattering potential is zero $\left\langle U^{3}\right\rangle=0$, then $\alpha_{s s}^{S H}=0,\langle\ldots\rangle$ denotes the ensemble average over roughness profiles. Therefore, if the distribution function of surface roughness taken to be Gaussian, the SS does not have any contribution in SHC induced by surface roughness. Contrarily, for that to be non-Gaussian, the SS can contribute in SHC because of $\left\langle U^{3}\right\rangle \neq 0$. The intrinsic SHC is negligible, i.e. $\sigma_{\text {int }}^{S H}=0$ as we consider the films with very weak bulk SOC such as $C u$. For calculation of $\alpha_{s j}^{S H}$, SHA due to SJ mechanism, we need to compute the velocity of an electron $\mathcal{V}_{n q}^{\sigma}$ in the presence of the spin-orbit potential $u_{R}^{S O}$. It can be found by calculating the matrix element $\mathcal{V}_{n q}^{\sigma}=\left\langle n q^{+} \sigma|\hat{\mathcal{V}}| n q^{+} \sigma\right\rangle$ of the velocity operator

$$
\hat{\mathcal{V}}=\frac{1}{i \hbar}[r, \mathcal{H}]=\frac{p}{m}+\frac{\eta}{\hbar}\left(\sigma \times \nabla u_{R}\right)
$$

between the scattering state $\left|n q^{+} \sigma\right\rangle=|n q \sigma\rangle+\sum_{n^{\prime} q^{\prime}} u_{q^{\prime} q}\left(\varepsilon_{n q}-\varepsilon_{n^{\prime} q^{\prime}}+i \epsilon\right)^{-1}\left|n^{\prime} q^{\prime} \sigma\right\rangle$ within 


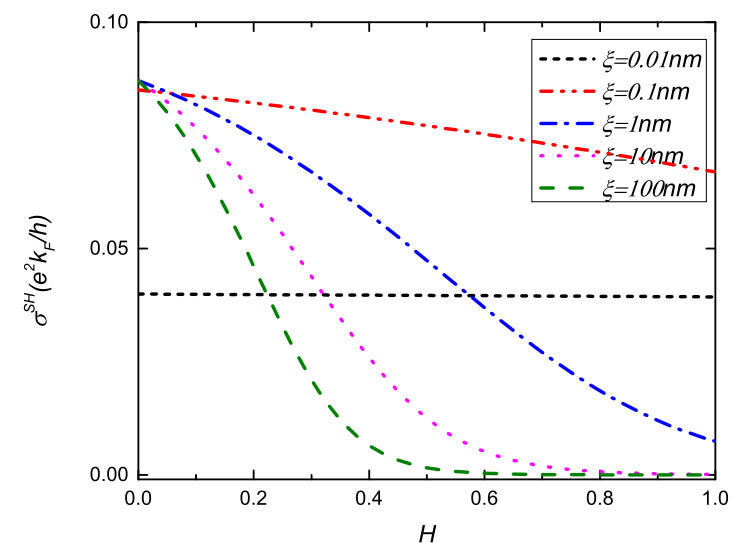

Figure 4. SHC $\sigma^{S H}$ as a function of Hurst exponent $H$ for $C u$ film with $\bar{\eta}=0.5$ and same parameters used in Fig, 1, and indicated values of $\xi$.

the Born approximation, and becomes

$$
\mathcal{V}_{n q}^{\sigma}=v_{n q}+\omega_{n q}^{\sigma}, \omega_{n q}^{\sigma}=\alpha_{s j}^{S H}\left(\sigma_{\sigma \sigma} \times v_{n q}\right),
$$

where $v_{n q}=\hbar q / m^{*}$ is the ordinary velocity, $\omega_{n q}^{\sigma}$ is the anomalous velocity, $\sigma_{\sigma \sigma}=\langle\sigma|\hat{\sigma}| \sigma\rangle$ is the polarization vector. SHA due to SJ is $\alpha_{s j}^{S H}=\frac{\hbar \eta}{2 \varepsilon_{F} \tau_{n}^{\prime}}$, by substituting of $\alpha_{s j}^{S H}$ in Eq. 13 . the SHC becomes,

$$
\sigma^{S H}=\frac{e^{2} k_{F}}{h} \frac{\bar{\eta}}{n_{c} \pi} \sum_{n=1}^{n_{c}} \frac{\tau_{n}}{\tau_{n}^{\prime}}\left(1-\frac{n^{2}}{n_{c}^{2}}\right),
$$

where $\bar{\eta}=k_{F}^{2} \eta$ and $h$ is the Planck's constant.

Therefor, we show that if distribution function of surface roughness to be Gaussian the SHE is only from the SJ contribution and for that to be non-Gaussian, both SJ and SS are present the SJ has the main contribution to the SHC.

\section{ROUGHNESS SURFACE MODEL}

In this section, we investigate the influence of uncorrelated and self-affine surface profiles on SHC. 


\section{A. Uncorrelated surface profile}

In this case, the surface roughness is uncorrelated and the correlation function and the its Fourier transform becomes,

$$
C(\rho)=\Lambda a_{0}^{2} \delta_{D}\left(\rho^{\prime}-\rho^{\prime \prime}\right)
$$

and

$$
\left\langle|S(q)|^{2}\right\rangle=a a_{0}^{2} \Lambda
$$

where $\Lambda=\left(\frac{\delta}{d}\right)^{2}$ is the dimensionless parameter, $\delta$ is variance of height fluctuations. The

normalization condition $\int_{0<q<q_{c}}\left\langle|S(q)|^{2}\right\rangle d^{2} q=\left(\frac{2 \pi}{a_{0}}\right)^{2} \Lambda$ yields the parameter $a$. This parameter is used to satisfy of the normalization condition for Fourier transform of correlation function. Here, $q_{c}=\pi / a_{0}$, is the upper cutoff in the Fourier space where $a_{0} \sim k_{F}^{-1}$ is the lattice constant. Surface scattering relaxation time $\tau_{n}^{\prime}$ obtained by substituting Eq.(18) in Eq.(11). By substituting $\tau_{n}^{\prime}$ in Eq.(12) and (16) the longitudinal conductivity and SHC can be obtained. The results agree with those found in Ref. 23.

\section{B. Self-affine fractal surface profile}

For a typical self-affine surface with power-law correlation function $C(\rho)$ is characterized by a correlation length $\xi^{29 \mid 30}$,

$$
C(\rho)=\Lambda\left(1-\left(\frac{\rho}{\xi}\right)^{2 H}\right)
$$

the roughness exponent $0 \leq H \leq 1$ is a measure of the degree of surface irregularity. Small values of $H$ characterize jagged or irregular surfaces at short length scales $(\rho<<\xi)$, where the correlation function shows power-law behavior, while large values of $H$ correspond to smoother height-height fluctuations. The Fourier transform of correlation for self-affine fractals has the scaling behavior if $q \xi>>1$ and for white noise profile that occurs if $q \xi<<1$. We use k-correlation model for description of self-affine surface,

$$
\left\langle|S(q)|^{2}\right\rangle=\frac{2 \pi}{a_{0}^{2}} \frac{\xi^{2} \Lambda}{\left(1+a q^{2} \xi^{2}\right)^{1+H}},
$$




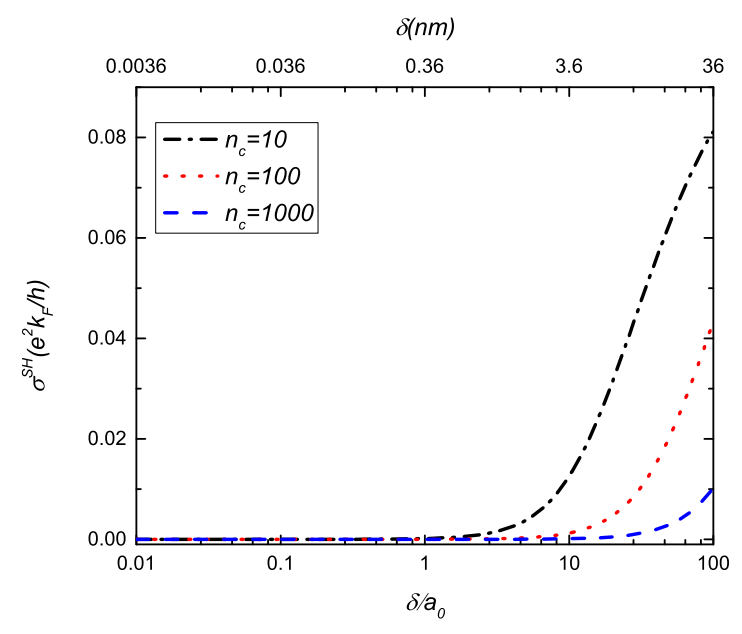

Figure 5. SHC $\sigma^{S H}$ for $C u$ thin film as a function of $\delta$ for three values of film thickness $n_{c}=$ $10,100,1000$ with $\varepsilon_{F}=7 \mathrm{eV}, q \sim k_{F}=1.361 / \AA, a_{0}=3.61 \AA, \sigma_{0}=5.88 \times 10^{7} \mathrm{~s} / \mathrm{m}, \tau_{0}=24 f \mathrm{~s}, \bar{\eta}=0.5$, $\xi=10 \mathrm{~nm}$ and $H=0.5$.

the normalization condition yields the parameter $a$,

$$
a=\left\{\begin{array}{ll}
(1 / 2 H)\left[1-\left(1+a q_{c}^{2} \xi^{2}\right)^{-H}\right] & 0<H \leq 1 \\
(1 / 2) \ln \left(1+a q_{c}^{2} \xi^{2}\right) & H=0
\end{array} .\right.
$$

By substituting Eq. 20) into Eq. (11) and by substituting $\tau_{n}^{\prime}$ into Eq.12 and (16) we are able to investigate the effect of fractal surface in SHC and SHA.

In Fig 3 we present the $\mathrm{SHC} \sigma^{S H}$, versus correlation length $\xi$ for several values of roughness exponent $H$. A characteristic feature seen in Fig. 3 is the presence of a maximum in the $\sigma^{S H}$ as function of the roughness exponent $H$, which occurs approximately at $\xi=0.1 n m \sim \mathcal{O}\left(a_{o}\right)$. For large values of $\xi$, the $\sigma^{S H}$ exhibits a normal behavior. It decreases with increasing $\xi$ or increasing $H$ (surface smoothing) and for small values of $\xi \ll 0.1 \mathrm{~nm}$ (in this case the correlation length is so smaller than the lattice constant, therefore we can suppose that the surface is uncorrelated $\xi \sim 0$.) the situation is reversed. This is due to the fact that this kind of roughness is in a range $q \xi<<1$. In this range, the power spectrum in power-law model is white noise and does not have scaling behavior. Also in large scale, in the logarithmic roughness $(H=0)$, the SHC increases extremely slowly with increasing correlation length. In Fig 4 for several values of correlation length we present the SHC as a function of roughness exponent $H$ for $C u$ thin film. Note that $\sigma^{S H}$ is larger for a rougher 


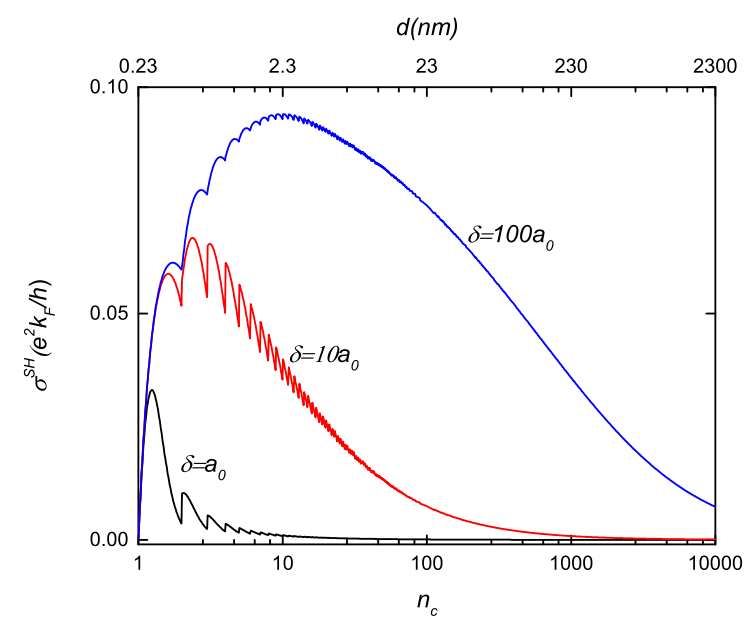

Figure 6. Spin Hall conductivity $\sigma^{S H}$ for $C u$ thin film as a function of $n_{c}$ with $\xi=1 \mathrm{~nm}$ and same parameters as in Fig,5, and indicated value of $\delta$.

surface with smaller correlation length.

The dependence of SHC on the $\delta$ and $n_{c}$ for $C u$ thin film with self-affine roughness profile is shown in Fig.5 and Fig.6. For $\delta / n c<a_{0}$ thin films the conductivity is independent on $\delta$ and for $\delta / n c>a_{0}$ the SHC increases with increasing ratio $\delta / n_{c}$, in agreement with those in Ref. 23. Also in Fig 6 the thickness dependence of SHC is plotted and is found to show oscillatory behavior dependent upon $\delta$ in $n c \sim 5-30$ because of quantum size effect $\frac{28}{\text {. In }}$ addition, the conductivity has a maximum in the dependence on $\delta$ then it decreases with increasing $n_{c}$.

Thus in the thinner and rougher film, $\sigma^{S H}$ is larger and at large scale of the correlation length $(d<<\xi)$ gets strongly influenced by the roughness exponent. The SHC and SHA can increase by one order of magnitude at large scale of the $\xi$ when the roughness exponent varies from $H=1$ to $H=0$. As shown in Fig.7 the SHA in large scale can be enhanced by (i) decreasing correlation length $\xi$, (ii) decreasing roughness exponent $H$ and in small scale the situation is reversed. Based on experiments, the general trends for the thin films is that the SHC increases with film roughness ${ }^{33 \mid 34}$ which seems to agree with our aforementioned argument. In general, one tends to use the interface width (root-mean square roughness) $\delta$ to measure how rough the surface is: if $\delta$ is large, then the surface is rougher. However, we have demonstrated that if the surface is self-affine the SHC and SHA depends not only on $\delta$ but also on the film thickness $d$, the lateral correlation length $\xi$, and the roughness exponent $H$. 

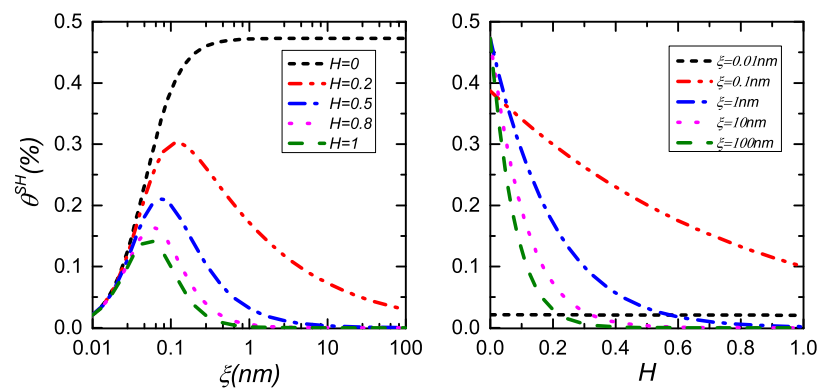

Figure 7. The SHA $\theta(\%)$ for $C u$ film with $n_{c}=100, \delta=5 a_{0}, \bar{\eta}=0.5$ and the same parameter used in Fig 1. Left panel shows the SHA as a function of correlation length $\xi$ and right panel shows the SHA as a function of Hurst exponent $H$.

\section{CONCLUSION}

In conclusion, we study the contribution of surface roughness and the fractality effects on the SHE of metallic thin films with self-affine correlation as a more realistic model. In the group of three surface roughness parameters $(\delta, \xi, H)$, major interplay of the roughness effect occurs for $H$ and $\xi$. The parameter $\delta$ has a minor effect (especially in thicker films) since it appears in the form a multiplication factor $\left(\sigma^{S H} \sim \delta^{-2}\right)$. The roughness exponent $H$ has a powerful impact on the SHC mainly for relatively large correlation lengths that can increase the SHA for $\mathrm{Cu}$ thin film one order of magnitude. The SHA due to fractal roughness scattering increases with reducing the correlation length and Hurst exponent and film thickness in large scale whereas the situation is reversed in small scale. Moreover, we found that if distribution function of surface roughness to be Gaussian the SHE is only from the SJ contribution and for that to be non-Gaussian, both SJ and SS are present. Our results uncover additional contribution from statistical information at surface of thin films which have important effects in the SHE.

* Corresponding author email address: m-mohseni@sbu.ac.ir, majidmohseni@gmail.com

1 M. Dyakonov and V. Perel, Physics Letters A 35, 459 (1971).

2 J. Hirsch, Physical Review Letters 83, 1834 (1999).

3 A. Hoffmann, IEEE Transactions on Magnetics 49, 5172 (2013). 
4 J. Sinova, S. O. Valenzuela, J. Wunderlich, C. Back, and T. Jungwirth, Reviews of Modern Physics 87, 1213 (2015).

5 Y. Niimi and Y. Otani, Reports on Progress in Physics 78, 124501 (2015).

6 S. Maekawa, S. O. Valenzuela, E. Saitoh, and T. Kimura, Spin Current, Vol. 17 (Oxford University Press, 2012).

7 Y. Kato, R. Myers, A. Gossard, and D. Awschalom, science 306, 1910 (2004).

8 J. Wunderlich, B. Kaestner, J. Sinova, and T. Jungwirth, Physical review letters 94, 047204 (2005).

9 E. Saitoh, M. Ueda, H. Miyajima, and G. Tatara, Applied Physics Letters 88, 182509 (2006).

10 S. O. Valenzuela and M. Tinkham, Nature 442, 176 (2006).

11 H. Zhao, E. J. Loren, H. Van Driel, and A. L. Smirl, Physical review letters 96, 246601 (2006).

12 I. M. Miron, K. Garello, G. Gaudin, P.-J. Zermatten, M. V. Costache, S. Auffret, S. Bandiera, B. Rodmacq, A. Schuhl, and P. Gambardella, Nature 476, 189 (2011).

13 L. Liu, C.-F. Pai, Y. Li, H. Tseng, D. Ralph, and R. Buhrman, Science 336, 555 (2012).

14 K. Uchida, J. Xiao, H. Adachi, J.-i. Ohe, S. Takahashi, J. Ieda, T. Ota, Y. Kajiwara, H. Umezawa, H. Kawai, et al., Nature materials 9, 894 (2010).

15 T. Jungwirth, J. Wunderlich, and K. Olejník, Nature materials 11, 382 (2012).

16 H. Nakayama, M. Althammer, Y.-T. Chen, K. Uchida, Y. Kajiwara, D. Kikuchi, T. Ohtani, S. Geprägs, M. Opel, S. Takahashi, et al., Physical review letters 110, 206601 (2013).

17 W. Zhang, W. Han, X. Jiang, S.-H. Yang, and S. S. Parkin, Nature Physics 11, 496 (2015).

18 T. Chen, R. K. Dumas, A. Eklund, P. K. Muduli, A. Houshang, A. A. Awad, P. Dürrenfeld, B. G. Malm, A. Rusu, and J. Åkerman, Proceedings of the IEEE 104, 1919 (2016).

19 J. Wunderlich, B.-G. Park, A. C. Irvine, L. P. Zârbo, E. Rozkotová, P. Nemec, V. Novák, J. Sinova, and T. Jungwirth, Science 330, 1801 (2010).

20 J. Wunderlich, A. Irvine, J. Sinova, B. Park, L. Zârbo, X. Xu, B. Kaestner, V. Novák, and T. Jungwirth, Nature Physics 5, 675 (2009).

21 K. Ando, M. Morikawa, T. Trypiniotis, Y. Fujikawa, C. Barnes, and E. Saitoh, Applied Physics Letters 96, 082502 (2010).

22 A. Kirihara, K.-i. Uchida, Y. Kajiwara, M. Ishida, Y. Nakamura, T. Manako, E. Saitoh, and S. Yorozu, Nature materials 11, 686 (2012). 
23 L. Zhou, V. L. Grigoryan, S. Maekawa, X. Wang, J. Xiao, et al., Physical Review B 91, 045407 (2015).

24 Y. Zhao, G.-C. Wang, and T.-M. Lu, Characterization of Amorphous and Crystalline Rough Surface-Principles and Applications, Vol. 37 (Academic press, 2000).

25 M. Jannesar, G. Jafari, S. V. Farahani, and S. Moradi, Thin Solid Films 562, 372 (2014).

26 G. Fishman and D. Calecki, Physical Review B 43, 11581 (1991).

27 Z. Tešanović, M. V. Jarić, and S. Maekawa, Physical review letters 57, 2760 (1986).

28 N. Trivedi and N. Ashcroft, Physical Review B 38, 12298 (1988).

29 G. Palasantzas and J. Barnaś, Physical Review B 56, 7726 (1997).

30 H. A. Makse, S. Havlin, M. Schwartz, and H. E. Stanley, Physical Review E 53, 5445 (1996).

31 M. Zamani, M. Salami, S. Fazeli, and G. Jafari, Journal of modern optics 59, 1448 (2012).

32 T. Jamali, S. V. Farahani, M. Jannesar, G. Palasantzas, and G. Jafari, Journal of Applied Physics 117, 175308 (2015).

33 M. Akyol, W. Jiang, G. Yu, Y. Fan, M. Gunes, A. Ekicibil, P. Khalili Amiri, and K. L. Wang, Applied Physics Letters 109, 022403 (2016).

34 H. An, Y. Kageyama, Y. Kanno, N. Enishi, and K. Ando, Nature communications 7 (2016). 